\title{
离子液体中哒嗪衍生物的高效、绿色合成方法研究
}

\author{
陈婷婷刘昆陈兆祥王媛媛* 戴立益* \\ (华东师范大学化学系 上海 200241)
}

\begin{abstract}
摘要 首次报道了以离子液体作为反应介质, 通过 1,2,4,5-四嗪与亲二烯体的逆 Diels-Alder环加成反应合成哒嗪衍生物 的方法. 与传统的合成方法相比, 以简便易得的传统咪唑离子液体兼作溶剂和催化剂, 反应时间从传统方法的 $13 \mathrm{~d}$ 缩 短为 $6 \mathrm{~h}$, 产物产率从 $64.0 \%$ 提高到 $85.5 \%$. 根据实验结果研究了离子液体与反应物之间的相互作用, 并提出可能的催 化机理. 该方法反应条件温和, 操作简便, 避免了有毒挥发性有机溶剂和额外催化剂的使用, 离子液体重复使用六次 未见产物产率明显降低, 更加符合绿色化学和原子经济性的要求.
\end{abstract}

关键词哒嗪衍生物; 1,2,4,5-四溙; 亲二烯体; 逆 Diels-Alder 反应; 离子液体

\section{An Efficient and Environmentally Friendly Procedure for Synthesis of Pyridazine Derivatives in lonic Liquids}

\author{
Chen, Tingting Liu, Kun Chen, Zhaoxiang Wang, Yuanyuan* Dai, Liyi* \\ (Department of Chemistry, East China Normal University, Shanghai 200241)
}

\begin{abstract}
Imidazolium ionic liquids have been investigated as reaction media in inverse-type Diels-Alder reactions between 1,2,4,5-tetrazine and a series of dienophiles to synthesize pyridazine derivatives for the first time. Comparing to conventional synthetic methods, using easily accessible imidazolium ionic liquids as solvent and catalyst, the reaction time reduced from $13 \mathrm{~d}$ to $6 \mathrm{~h}$ and product yields increased from $64.0 \%$ to $85.5 \%$. The interaction between ionic liquid and reactants in theoretical way was further researched and the most possible catalytic mechanism was proposed. The reaction condition is mild and easy to operate, avoiding using the volatile organic solvents and additional catalysts. Moreover, ionic liquids can be used for more than six times without obvious loss of product yields. Therefore, the current synthetic approach is more in accordance with green chemistry and atom economy requirements.
\end{abstract}

Keywords pyridazine derivative; 1,2,4,5-tetrazine; dienophile; inverse-type Diels-Alder reaction; imidazolium ionic liquid

取代或者功能化的哒嗪在无机、有机和药物化学领 域发挥着重要作用 ${ }^{[1]}$. 作为药用活性化合物的结构成分, 哒嗪环也是多巴胺能、胆碱能、类胆碱能和金属配合物 的组成元素 ${ }^{[2,3]}$. 此外, 哒嗪还是构建其他杂环化合物 ${ }^{[4]}$ 和物理有机化学 ${ }^{[5]}$ 中的重要中间体, 其在肽产业中也被 作为一种新型 $\alpha$-螺旋模拟物进行探索 ${ }^{[6]}$. 考虑到众多哒 嗪衍生物的广泛应用价值, 构建经济、简洁而高效地合 成哒嗪衍生物的方法有重要的研究意义. 已报道的合成 方法包括经典的杂环形成反应、嵌入式环缩合反应、分 子内缩合 ${ }^{[7,8]}$ 和环加成反应. 其中, $1,2,4,5$-四溙和富电子 的亲二烯体的反应是发展的最为成熟的逆 Diels-Alder 反应. 然而, 目前的研究方法仍有部分缺点叒待完善,
如反应条件苛刻、操作不便、工艺复杂 ${ }^{[9]}$ 、使用微波等 替代能源 ${ }^{[10]}$ 以及产物产率低等, 这些都为工业化生产 带来困难. 最近, 谢合新等 ${ }^{[11]}$ 提出了一种通过脯氨酸催 化酮和 1,2,4,5-四嗪反应直接合成哒嗪的方法. 这种方 法无疑给合成哒嗪带来了新见解，但是该合成方法使用 了大量的有机溶剂, 并有一定量的副产物生成, 这些都 不符合原子经济性理念的要求.

由于具有优异的物理化学性质 ${ }^{[12,13]}$, 近年来, 离子 液体作为 “绿色介质” 代替挥发性有机溶剂或有毒催化 剂, 在有机合成反应中得到广泛应用. 此外, 随着离子 液体特别是咪唑类离子液体的大规模商业化生产，离子 液体在有机合成反应中的应用也更经济可行. 前期的研

\footnotetext{
* E-mail: yywang@chem.ecnu.edu.cn, lydai@chem.ecnu.edu.cn

Received October 31, 2013; revised January 8, 2013; published online January 10, 2014

Project supported by the National Natural Science Foundation of China (Nos. 21003049, 21073064).

国家自然科学基金(Nos. 21003049, 21073064 )资助项目.
} 
究已表明, 溶剂和催化剂可以显著影响逆 Diels-Alder 反 应的进行. 将离子液体作为反应介质应用于逆 DielsAlder 反应中，可克服上述传统哒嗪衍生物合成方法的 缺点. 但是, 目前很少有离子液体在哒溙衍生物合成反 应中应用的报道.

我们课题组 ${ }^{[14 ~ 16]}$ 长期以来致力于离子液体中绿色 有机合成反应的研究. 作为前期工作的延续, 此次我们 选取了七种代表性的离子液体，1-丁基-3-甲基咪唑溴盐 ([Bmim]Br)、1-丁基 3-甲基咪唑四氟硼酸盐([Bmim]$\mathrm{BF}_{4}$ )、1-丁基-3-甲基咪唑六氟磷酸盐([Bmim $\left.] \mathrm{PF}_{6}\right) 、 1$ 丁基-3-甲基咪唑硫酸氢盐([Bmim] $\mathrm{HSO}_{4}$ )、1-丁基-2,3二甲基咪唑溴盐([ $\left.\left.\mathrm{Bm}_{2} \mathrm{im}\right] \mathrm{Br}\right) 、 1$-丁基吡啶溴盐([Bp] $\left.\mathrm{Br}\right)$ 和 1-丁基-2-羟甲基-3-甲基咪唑溴盐([Bhmim] Br)作为溶 剂和催化剂, 考察其在 1,2,4,5-四嗪和一系列亲二烯体 的环加成反应中的应用. 实验结果表明: 1-丁基-3-甲基 咪唑阳离子的离子液体能显著加快反应速率(反应时间 从 $13 \mathrm{~d}$ 缩短至 $6 \mathrm{~h}$ ) 和改进产物的产率(由 $64.0 \%$ 提高到 $85.5 \%$ ). 离子液体使用六次以后，仍能保持良好的性能, 产物产率没有明显的降低. 此外, 我们还进一步研究了 离子液体在哒嗪衍生物合成反应过程中的作用, 并提出 可能的催化机理.

\section{1 结果与讨论}

Sauer 等 ${ }^{[17]}$ 曾报道过 $1,2,4,5$-四嗪与苯乙炔在传统有 机溶剂中 1,4-二氧六环里的反应, 产率为 $64.0 \%$ (表 1 , 序号 1). 我们也分别研究了 $1,2,4,5$-四嗪与苯乙炔在六 种不同离子液体中的产率, 以做比较. 从表 1 可以发现, 与 1,4-二氧六环相比, 1-丁基-3-甲基咪唑阳离子的离子 液体和[Bhmim] Br (Table 1, Entries 2 5, 7)中产物的产 率提高了近 $30 \%$, 反应速率大大提高, 反应时间从 $13 \mathrm{~d}$ 缩短为 $6 \mathrm{~h}$. 而离子液体 $\left[\mathrm{Bm}_{2} \mathrm{im}\right] \mathrm{Br}$ 和 $[\mathrm{Bp}] \mathrm{Br}$ 中的产率相 对较低, 分别为 78.2\%和 62.5\%. 我们认为 1-丁基-3-甲 基咪唑阳离子的离子液体在反应中发挥了溶剂和 Lewis 酸催化剂的作用, 即它们的阳离子 $[\mathrm{Bmim}]^{+}$二号位碳上 的氢原子与四嗪形成氢键, $[\mathrm{Bhmim}] \mathrm{Br}$ 中的羟甲基也能 与四溙形成氢键, 降低了反应能量, 而 $\left[\mathrm{Bm}_{2} \mathrm{im}\right] \mathrm{Br}$ 中二 号碳上的氢原子被甲基所取代, 四五号碳上的氢原子与 四嗪形成氢键的能力相比二号碳上的氢较弱, 所以产率 有所降低, [Bp]Br 的阳离子无法与四嗪形成氢键, 它仅 起到极性溶剂的作用, 故反应产率最低. 这也与先前文 献的报道结果相符 ${ }^{[18]}$. 1-丁基-3-甲基咪唑阳离子的离子 液体中, $\left[\mathrm{Bmim}^{-} \mathrm{PF}_{6}\right.$ 具有疏水性, 这给收集产物和回收 离子液体带来困难; $[\mathrm{Bmim}] \mathrm{BF}_{4}$ 和 $[\mathrm{Bmim}] \mathrm{HSO}_{4}$ 的合成 均是从中间体 $[\mathrm{Bmim}] \mathrm{Br}$ 经过阴离子交换然后进一步纯 化得到, [Bhmim] $\mathrm{Br}$ 也是在 $[\mathrm{Bmim}] \mathrm{Br}$ 的基础上与多聚甲
醛反应制得的，这些都增加了离子液体的合成成本. 故 我们选取一步即可制备的 [Bmim] $\mathrm{Br}$ 为 1,2,4,5-四嗪和苯 乙炔反应的标准溶剂, 进一步优化反应条件. 其他条件 相同时，离子液体的使用量不同，反应产率也有明显的 变化(表 1, 序号 5 7). [Bmim] $\mathrm{Br}$ 的使用量为 $5 \mathrm{mmol}$ 时, 产物产率反而降低了, 这是因为过量的离子液体会与 1,2,4,5-四嗪形成氢键, 降低了 $1,2,4,5$-四嗪的浓度 ${ }^{[19]}$. 因此离子液体的最佳用量为 $3 \mathrm{mmol}$.

表 1 不同离子液体对 1,2,4,5-四嗪和苯乙炔反应的影响 Table 1 Effect of different ionic liquids (Ils) in the reaction of 1,2,4,5-tetrazinewith phenylacetylene

\begin{tabular}{cccccc}
\hline Entry & Solvent & $\begin{array}{c}\text { Quantity of } \\
\text { IL/mmol }\end{array}$ & Time & $\begin{array}{c}\text { Yield of } \\
\mathbf{3 a}^{b} / \%\end{array}$ & Selectivity/\% \\
\hline 1 & $1,4-\mathrm{Dioxane}$ & - & $13 \mathrm{~d}$ & 64.0 & 100 \\
2 & {$\left[\mathrm{Bmim}_{\mathrm{B}} \mathrm{BF}_{4}\right.$} & 3 & $6 \mathrm{~h}$ & 86.3 & 100 \\
3 & {$\left[\mathrm{Bmim}_{\mathrm{PF}_{6}}\right.$} & 3 & $6 \mathrm{~h}$ & 87.9 & 100 \\
4 & {$[\mathrm{Bmim}] \mathrm{HSO}_{4}$} & 3 & $6 \mathrm{~h}$ & 86.6 & 100 \\
5 & {$\left[\mathrm{Bmim}_{\mathrm{Br}}\right.$} & 3 & $6 \mathrm{~h}$ & 85.5 & 100 \\
6 & {$\left[\mathrm{Bm}_{2} \mathrm{im}\right] \mathrm{Br}$} & 3 & $6 \mathrm{~h}$ & 78.2 & 100 \\
7 & {$[\mathrm{Bhmim}] \mathrm{Br}$} & 3 & $6 \mathrm{~h}$ & 89.0 & 100 \\
8 & {$[\mathrm{Bp}] \mathrm{Br}$} & 3 & $6 \mathrm{~h}$ & 62.5 & 100 \\
9 & {$[\mathrm{Bmim}] \mathrm{Br}$} & 5 & $6 \mathrm{~h}$ & 84.1 & 100 \\
10 & {$[\mathrm{Bmim}] \mathrm{Br}$} & 1 & $6 \mathrm{~h}$ & 50.4 & 100 \\
\hline
\end{tabular}

图 1A 展示了温度对反应产率的影响. 从图中可以 观察到: 在 343 363 K 的范围内, 产物的产率随着温度 的提高迅速上升, 继续升高温度, 产率开始下降. 造成 这一趋势的原因可能是：过高的温度会加速 1,2,4,5-四 嗪的挥发, 从而降低反应物的实际浓度，降低了产率. $363 \mathrm{~K}$ 时产率达到最大值为 $85.5 \%$, 所以最佳的反应温 度为 $363 \mathrm{~K}$.

反应时间对产率也有显著的影响，特别是在反应的 起始阶段. 如图 1B 所示, 反应 $6 \mathrm{~h}$ 之前，由于反应物的 浓度较高，随着时间的增加反应产率也在高速增加. $6 \mathrm{~h}$ 后, 产率基本保持不变. 这表明该环加成反应在 $6 \mathrm{~h}$ 达 到反应平衡态. 从节省能源的角度考虑，反应的最佳时 间为 $6 \mathrm{~h}$.

我们还考察了其他条件相同时, $1,2,4,5$-四嗪与苯乙 炔的摩尔比对产物产率的影响, 结果如图 1C 所示. 显 然，1,2,4,5-四嗪与苯乙炔的物质的量比对产物产率具有 很大的影响. 在 1 到 2 范围内改变 1,2,4,5-四溙与苯乙炔 的物质的量比, 产物的产率起初逐渐上升然后下降，曲 线的顶点位于物质的量比为 1.5 时. 从理论上而言, 1,2,4,5-四嗪与苯乙炔应按照 $1: 1$ 的物质的量比进行反 应，实际上物质的量比为 1 时，反应的产率仅为 $42.6 \%$. 这是因为 1,2,4,5-四嗪极易挥发, 实际物质的量比小于 1. 然而加入过多量的 $1,2,4,5$-四嗪, 即物质的量比超过 

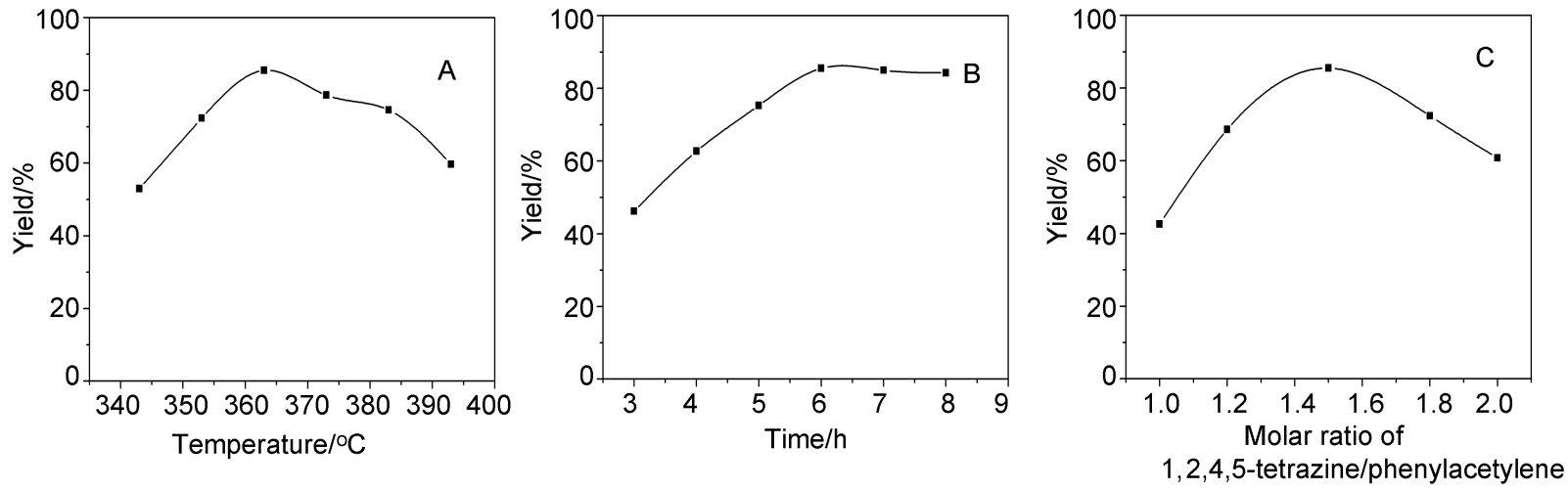

图 1 (A)温度、(B)时间和(C) 1,2,4,5-四嗪与苯乙炔的物质的量比对反应产率的影响

Figure 1 Effect of (A) temperature, (B) time and (C) initial molar ratio of 1,2,4,5-tetrazine/phenylacetylene on product yield

1.5 时, 额外的 $1,2,4,5$-四嗪就会与溶剂兼催化剂 $[\mathrm{Bmim}] \mathrm{Br}$ 形成氢 键 ${ }^{[19]}$, 这将降低溶剂兼催化剂 $[\mathrm{Bmim}] \mathrm{Br}$ 的浓度, 而且四嗪浓度过高也会降低另一反 应物苯乙炔的相对浓度, 从而降低反应产率. 综合考虑 $1,2,4,5$-四嗪与苯乙炔的最佳物质的量比为 1.5 .

为了扩大该反应的使用范围提高其普适性, 我们又 研究了多种不同亲二烯体在 $[\mathrm{Bmim}] \mathrm{Br}$ 中与 $1,2,4,5$-四嗪 反应制备哒嗪衍生物的反应. 如表 2 所示: 1,2,4,5-四嗪 与带有不同官能团的炔类和烯类亲二烯体反应均能获 得 $72.4 \% \sim 85.5 \%$ 的产率. 从实验数据可知，电子效应和 取代效应对反应产率有影响. 1,2,4,5-四嗪是缺电子化合 物，因此富电子的炔类和烯类化合物是该反应的良好供 体. 理论上讲, 携带供电子基团如甲氧基和甲基的炔类 化合物的产率应高于未取代的苯乙炔或带有缺电子基 团如硝基、氯原子的化合物, 然而苯乙炔的产率却是最 高的为 $85.5 \%$. 原因是以较大基团取代氢原子后将会产 生空间位阻效应. 我们的研究结果(表 2, 序号 1,3,5)还 表明，该反应中空间位阻效应大于电子效应，占据主导 地位. 此外, 作为烯类亲二烯体的乙氧基乙烯在很短的 反应时间里也可以获得 $81.6 \%$ 的产率. 上述反应的所有 产物的结构都已经过核磁 ${ }^{1} \mathrm{H}$ NMR 验证.

表 $2[\mathrm{Bmim}] \mathrm{Br}$ 对 1,2,4,5-四嗪和亲二烯体反应的普适性

Table 2 The generality of [Bmim]Br towards cycloadditions of 1,2,4,5-tetrazine to dienophiles

\begin{tabular}{ccccc}
\hline Entry & Dienophiles & Reaction time/h & Yield/\% & Selectivity $/ \%$ \\
\hline 1 & 2a & 6 & 85.5 & 100 \\
2 & 2b & 4 & 73.8 & 100 \\
3 & 2c & 5 & 82.9 & 100 \\
4 & 2d & 6 & 72.4 & 100 \\
5 & 2e & 6 & 75.2 & 100 \\
6 & 2f & 0.5 & 81.6 & 100 \\
\hline
\end{tabular}

Reaction conditions: 9 mmol of 1,2,4,5-tetrazine 1, $6 \mathrm{mmol}$ of dienophiles, 3 mmol of [Bmim] Br, $363 \mathrm{~K}$.

从经济和环境的角度考虑, 催化剂或溶剂的可回收
利用非常重要．与传统有机溶剂相比，易于回收并循环 使用是离子液体的优势之一. 因此, 我们研究了离子液 体 $[\mathrm{Bmim}] \mathrm{Br}$ 在该反应中的可回收性及回收效果. 反应 结束后, 将反应液冷却到室温并加入二氯甲烷和水进行 萃取. 将离子液体相从有机层分离出来, 然后在 $343 \mathrm{~K}$ 真空干燥 $2 \mathrm{~h}$. 根据表 3 结果可知, [Bmim]Br 经回收使用 六次后, 产物产率仍无显著的下降. 因此, $[\mathrm{Bmim}] \mathrm{Br}$ 是 $1,2,4,5$-四嗪和苯乙炔反应的一种高效可回收的溶剂和 催化剂.

表 3 [Bmim]Br 的回收使用情况

Table 3 Reusability of [Bmim] $\mathrm{Br}$

\begin{tabular}{cccc}
\hline Entry & Recycling & Yield $^{a} / \%$ of 3a & Selectivity $\%$ \\
\hline 1 & 0 & 85.5 & 100 \\
2 & 1 & 84.6 & 100 \\
3 & 2 & 83.9 & 100 \\
4 & 3 & 83.2 & 100 \\
5 & 4 & 82.8 & 100 \\
6 & 5 & 82.3 & 100 \\
7 & 6 & 81.5 & 100 \\
\hline
\end{tabular}

1,2,4,5-四嗪和苯乙炔是逆电子效应的 $[4+2]$ 环加成 反应, 该反应是由二烯体最低未占据轨道和亲二烯体最 高占有轨道共同控制的 ${ }^{[17]}$. [Bmim] Br 作为一种潜在的 氢键供体, 在反应过程中它可以通过咪唑环二号碳上的 氢原子与 $1,2,4,5$-四嗪形成氢键. 将 $1,2,4,5$-四溙和 $[\mathrm{Bmim}] \mathrm{Br}$ 以物质的量 $1: 1$ 混合后直接打核磁, ${ }^{1} \mathrm{H}$ NMR 谱图见图 2.1,2,4,5-四嗪存在时, 咪唑环二号碳氢的化 学位移由原来的 $\delta 10.323$ 移动到 $\delta 10.243(\delta 10.353$ 是四 嗪中两个氢的化学位移), 这是因为与 $1,2,4,5$-四嗪相比, 溴离子是更好的氢键受体, $[\mathrm{Bmim}]^{+}$与 $1,2,4,5$-四嗪形成 的氢键没有与溴离子的氢键强烈, 所以咪唑环二号碳氢 的化学位移向高场低位移方向移动. 类似地研究高国华 课题组 ${ }^{[20]}$ 也报道过。鉴于上述理论验证，本课题如 Scheme 1 所示. [Bmim] Br 与 1,2,4,5-四嗪形成的氢键可 
以有效降低二烯体的最低末占用轨道的能量从而缩小 它与亲二烯体最高占有轨道的能量差; 根据前线轨道理 论(FMO)这有利于增大环加成反应的速率常数最终提 高反应产率 ${ }^{[21]}$.

\section{2 结论}

本文选用简便易得的传统咪唑离子液体作为反应 介质, 高效且环境友好地实现了 1,2,4,5-四嗪与多种亲 二烯体的环加成反应. 与传统反应相比, 离子液体兼作 溶剂和催化剂, 反应时间大大缩短, 产物产率明显提高. 反应条件温和, 操作简便, 避免了有毒挥发性有机溶剂 和额外催化剂的使用, 且离子液体可以很好地回收重复 使用, 更加符合绿色化学和原子经济性的要求, 有助于 推进哒嗪衍生物的工业化生产进程.

\section{3 实验部分}

\section{1 仪器与试剂}

苯乙炔、4-硝基苯乙炔、4-甲氧基苯乙炔、4-氯苯 乙炔、4-甲基苯乙炔和乙氧基苯乙炔均从国药集团化学 试剂有限公司购买并直接使用. 离子液体 $[\mathrm{Bmim}] \mathrm{Br}$, $[\mathrm{Bmim}] \mathrm{BF}_{4},[\mathrm{Bmim}] \mathrm{PF}_{6},[\mathrm{Bmim}] \mathrm{HSO}_{4},\left[\mathrm{Bm}_{2} \mathrm{im}\right] \mathrm{Br},[\mathrm{Bp}]-$ $\mathrm{Br}$ 和化合物 1 (Scheme 2) 分别根据文献 $\left.{ }^{[22} 26\right]$ 制备. 使用 Varian-300 光谱仪测定 ${ }^{1} \mathrm{H}$ NMR 谱, 均采用 $\mathrm{CDCl}_{3}$ 作为 溶剂，化学位移以四甲基亚硅(TMS)为标准. 反应物的 转换率通过薄层色谱(TLC)进行检测, 所有的产率均是 分离产率.

\section{2 实验方法}

1,2,4,5-四嗪 $(9 \mathrm{mmol})$, 亲二烯体 $\mathbf{2} \mathbf{a} \sim \mathbf{2} \mathbf{f}(6 \mathrm{mmol})$ 和 离子液体(IL, $3 \mathrm{mmol}$ )分别加入到 $50 \mathrm{~mL}$ 的封管中，在

(a)

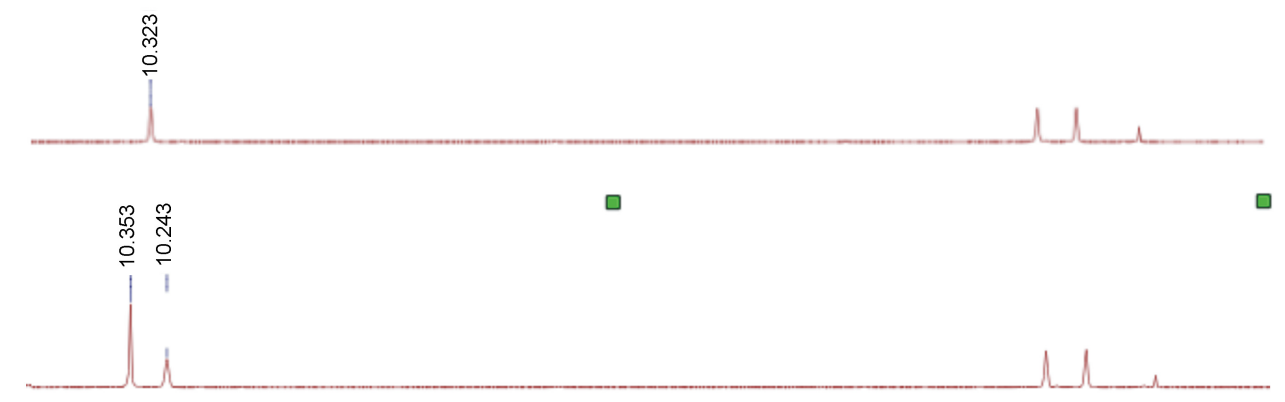

\begin{tabular}{lllllllllll}
\hline 10.5 & 10.2 & 9.8 & 9.4 & 9.0 & 8.6 & 8.2 & 7.8 & 7.4 & 7.0 \\
& & & $\delta$ & & & & &
\end{tabular}

图 $2[\mathrm{Bmim}] \mathrm{Br}$ 的 ${ }^{1} \mathrm{H}$ NMR 化学位移

Figure 2 Chemical shift in the ${ }^{1} \mathrm{H}$ NMR of [Bmim]Br

Solvent: $\mathrm{CDCl}_{3}$. (a) $[\mathrm{Bmim}] \mathrm{Br}$; (b) $n([\mathrm{Bmim}] \mathrm{Br}): n(1,2,4,5$-tetrazine $)=1: 1$
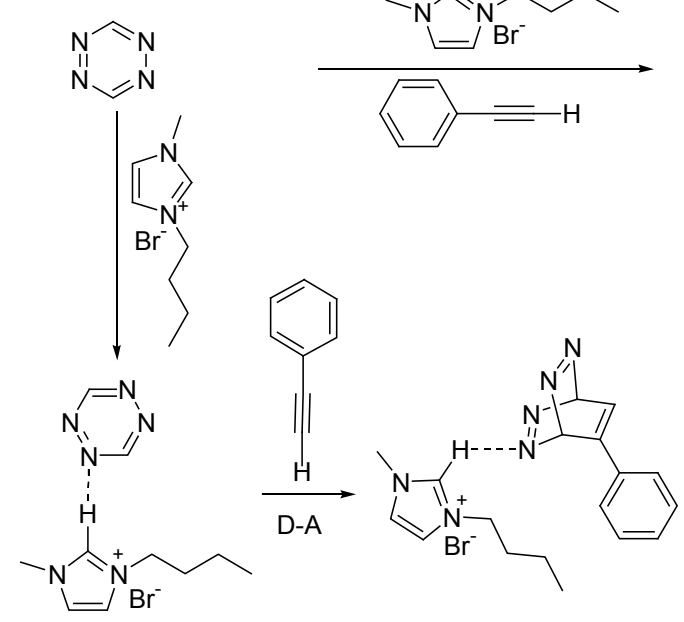

Scheme 1

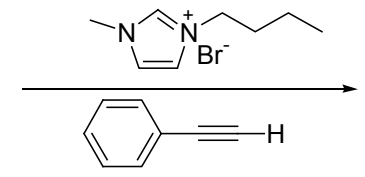<smiles></smiles>

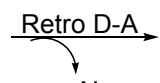<smiles></smiles> 
<smiles>c1nncnn1</smiles>

1

Scheme 2

给定温度下加热并持续搅拌直到反应结束为止(反应原 理, Scheme 3). 反应进程通过薄层色谱(TLC)来指示, 以 二氯甲烷和甲醇的体积比为 $20: 1$ 作为洗脱剂. 反应结 束后, 反应液用二氯甲烷 $(25 \mathrm{~mL})$ 萃取, 然后再用蒸馏水 $(10 \mathrm{~mL})$ 洗涤数次. 将从水层中分离出的有机相进行真 空旋蒸, 然后再经过油葲进一步纯化以期获得纯净的产 物. 通过核磁 ${ }^{1} \mathrm{H}$ NMR 谱来验证产物的结构, 表征数据 均与文献[17]报道值一致. 水相里的离子液体在 $343 \mathrm{~K}$ 真空下干燥 $2 \mathrm{~h}$, 即可回收利用并直接用于下一轮的实 验中.

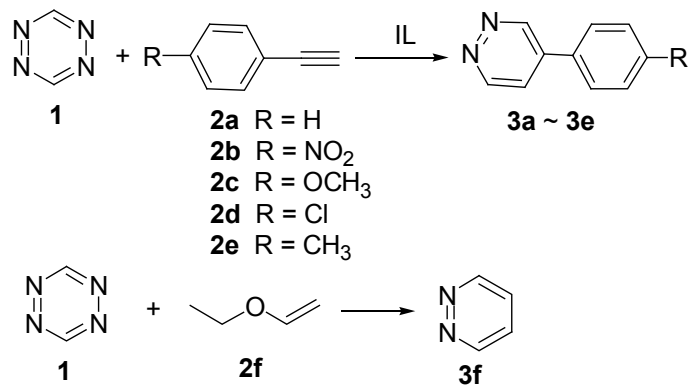

Scheme 3

3a: ${ }^{1} \mathrm{H}$ NMR $\left(300 \mathrm{MHz}, \mathrm{CDCl}_{3}\right) \delta: 9.45(\mathrm{dd}, J=2.2$, $1.0 \mathrm{~Hz}, 1 \mathrm{H}), 9.21(\mathrm{dd}, J=5.5,1.0 \mathrm{~Hz}, 1 \mathrm{H}), 7.60 \sim 7.70(\mathrm{~m}$, $3 \mathrm{H}), 7.45 \sim 7.59(\mathrm{~m}, 3 \mathrm{H})$.

3b: ${ }^{1} \mathrm{H}$ NMR $\left(300 \mathrm{MHz}, \mathrm{CDCl}_{3}\right) \delta: 9.50(\mathrm{~s}, 1 \mathrm{H}), 9.34$ $(\mathrm{d}, J=5.4 \mathrm{~Hz}, 1 \mathrm{H}), 8.42(\mathrm{~d}, J=7.1 \mathrm{~Hz}, 2 \mathrm{H}), 7.85(\mathrm{~d}, J=$ $8.6 \mathrm{~Hz}, 2 \mathrm{H})$.

3c: ${ }^{1} \mathrm{H}$ NMR $\left(300 \mathrm{MHz}, \mathrm{CDCl}_{3}\right) \delta: 9.44(\mathrm{~d}, J=1.1$ $\mathrm{Hz}, 1 \mathrm{H}), 9.16$ (d, $J=6.3 \mathrm{~Hz}, 1 \mathrm{H}), 7.65$ (d, $J=8.8 \mathrm{~Hz}, 2 \mathrm{H})$, $7.60(\mathrm{dd}, J=5.4,2.5 \mathrm{~Hz}, 1 \mathrm{H}), 7.06(\mathrm{~d}, J=8.8 \mathrm{~Hz}, 2 \mathrm{H})$, $3.89(\mathrm{~s}, 3 \mathrm{H})$.

3d: ${ }^{1} \mathrm{H}$ NMR $\left(300 \mathrm{MHz}, \mathrm{CDCl}_{3}\right) \delta: 9.45(\mathrm{~s}, 1 \mathrm{H}), 9.25$ $(\mathrm{d}, J=5.4 \mathrm{~Hz}, 1 \mathrm{H}), 7.62$ (dd, $J=5.6,3.0 \mathrm{~Hz}, 3 \mathrm{H}), 7.53$ (d, $J=8.5 \mathrm{~Hz}, 2 \mathrm{H})$.

3e: ${ }^{1} \mathrm{H}$ NMR $\left(300 \mathrm{MHz}, \mathrm{CDCl}_{3}\right) \delta: 9.45(\mathrm{~d}, J=1.2$ Hz, 1H), 9.19 (dd, $J=5.4,1.0 \mathrm{~Hz}, 1 \mathrm{H}), 7.64$ (dd, $J=5.4$,
$2.5 \mathrm{~Hz}, 1 \mathrm{H}), 7.58$ (d, $J=8.1 \mathrm{~Hz}, 2 \mathrm{H}), 7.35$ (d, $J=7.9 \mathrm{~Hz}$, $2 \mathrm{H}), 2.44(\mathrm{~s}, 3 \mathrm{H})$.

3f: ${ }^{1} \mathrm{H}$ NMR $\left(300 \mathrm{MHz}, \mathrm{CDCl}_{3}\right) \delta: 9.23(\mathrm{t}, J=3.5 \mathrm{~Hz}$, $2 \mathrm{H}), 7.65(\mathrm{t}, J=3.5 \mathrm{~Hz}, 2 \mathrm{H})$.

\section{References}

[1] Boger, D. L.; Patel, M. Prog. Heterocycl. Chem. 1989, 1, 36.

[2] (a) Wermuth, C. G. Heterocycl. Chem. 1998, 35, 1091

(b) Xiong, J. R.; Xiang, Q. X.; Chen, J. X.; Zhang, Y. Q. Chin. J. Org. Chem. 2008, 28, 137 (in Chinese). (熊俊如, 向清祥, 陈稼轩, 张元勤, 有机化学, 2008, 28, 137.)

[3] Xiong, J. R.; Xiang, Q. X. Chin. J. Org. Chem. 2006, 26, 487 (in Chinese). (熊俊如, 向清祥, 有机化学, 2006, 26,487).

[4] Naud, S.; Pipelier, M.; Viault, G. Eur. J. Org. Chem. 2007, 20, 3296.

[5] Yu, Z. X.; Dang, Q.; Wu, Y. D. J. Org. Chem. 2001, 66, 6029.

[6] Mann, E.; Moisan, L.; Hou, J. L. Tetrahedron Lett. 2008, 49, 903.

[7] Eicher, T.; Hauptmann, S.; Speicher, A. The Chemistry of Heterocycles—structure, Reactions, Synthesis, and Applications, Wiley$\mathrm{VCH}$, Weinheim, 2012, p. 512

[8] Joule,J. A.; Mills, K. Heterocyclic Chemistry, Wiley-Blackwell, United Kingdom, 2010, p. 236.

[9] Bourotte, M.; Pellegrini, N.; Schmitt, M. Synlett 2003, 1482.

[10] Hoogenboom, R.; Moore, B. C.; Schubert, U. S. J. Org. Chem. 2006, 71, 4903.

[11] Xie, H.; Zu, L.; Oueis, H. R. Org. Lett. 2008, 10, 1923.

[12] Ali, Z. H.; Samira, D.; Shahab, A. Fluid Phase Equilib. 2013, 360, 139.

[13] Brennecke, J. F.; Maginn, E. J. AIChE J. 2001, 47, 2384.

[14] Li, W.; Wang, Y.; Dai, L. Y. Catal. Lett. 2011, 141, 1651.

[15] Wang, Y. Y.; Zhou, J. L.; Liu, K. J. Mol. Catal. A: Chem. 2013, 366, 195.

[16] Wang, Y. Y.; Jiang, D.; Dai, L. Y. Catal. Commun. 2008, 9, 2475.

[17] Sauer, J.; Dieter, K.; Hetzenegger, H. J. Eur. J. Org. Chem. 1998, 2885.

[18] Ahmat, X.; Keyume, A.; Shinozaki, H. Chem. Res. Chin. Univ. 2009, 25, 161.

[19] Chiappe, C.; Malvaldi, M.; Pomelli, C. S. Green Chem. 2010, 12, 1330.

[20] Zhang, L. H.; Fu, X.; Gao, G.. H. ChemCatChem 2011, 3, 1359.

[21] Sauer, J. In Comprehensive Heterocyclic Chemistry II, Eds: Katritzky, A. R.; Rees, C. W.; Scriven, E. F. V., Pergamom Press, Oxford, 1996, p. 198.

[22] Fleming, I. Molecular Orbitals and Organic Chemical Reactions, Wiley, Chichester, UK, 2010, p. 336.

[23] Wang, X. S.; Zhang, M. M.; Jiang, H. Tetrahedron 2007, 63, 4439.

[24] Dubreuil, J. F.; Bourahlak; Rahmovni, M. Catal. Commun. 2002, 3, 185.

[25] Peng, Y.; Song, G. Catal. Commun. 2007, 8, 111.

[26] Tadeuse, B.; Przemyslaw, K. J. Polym. Sci., Polym. Chem. 2008, $46,6961$. 